\title{
Training Recurrent Networks
}

\section{Pedersen, Morten With}

Published in:

IEEE Workshop on Neural Networks for Signal Processing VII

Link to article, DOI:

10.1109/NNSP.1997.622416

Publication date:

1997

\section{Document Version}

Publisher's PDF, also known as Version of record

Link back to DTU Orbit

Citation (APA):

Pedersen, M. W. (1997). Training Recurrent Networks. In IEEE Workshop on Neural Networks for Signal Processing VII (pp. 355-364). IEEE. https://doi.org/10.1109/NNSP.1997.622416

\section{General rights}

Copyright and moral rights for the publications made accessible in the public portal are retained by the authors and/or other copyright owners and it is a condition of accessing publications that users recognise and abide by the legal requirements associated with these rights.

- Users may download and print one copy of any publication from the public portal for the purpose of private study or research.

- You may not further distribute the material or use it for any profit-making activity or commercial gain

- You may freely distribute the URL identifying the publication in the public portal

If you believe that this document breaches copyright please contact us providing details, and we will remove access to the work immediately and investigate your claim. 


\title{
TRAINING RECURRENT NETWORKS
}

\author{
Morten With Pedersen \\ CONNECT, Department of Mathematical Modelling, Building 321 \\ Technical University of Denmark, DK-2800 Lyngby, Denmark \\ Phone: +4545253920 Fax: + 4545872599 \\ email: mwp@imm.dtu.dk
}

\begin{abstract}
Training recurrent networks is generally believed to be a difficult task. Excessive training times and lack of convergence to an acceptable solution are frequently reported. In this paper we seek to explain the reason for this from a numerical point of view and show how to avoid problems when training. In particular we investigate illconditioning, the need for and effect of regularization and illustrate the superiority of second-order methods for training.
\end{abstract}

\section{INTRODUCTION}

Recurrent neural networks are an interesting class of models for signal processing as they are able to build up internal memory suited for the task at hand and thus often lead to compact model representations. However, it is generally believed to be a difficult task to train this type of networks. Several authors have addressed the learning problem for recurrent networks, e.g., in the context of sequence classification when required to store information for an arbitrary period of time $[1,5]$ but to the best of the authors knowledge no one have treated the problem from a general numerical point of view.

Feedforward networks were treated extensively from a numerical point of view in [7] where it was illustrated how training forms an extremely illconditioned optimization problem. In this contribution we extend this analysis to include recurrent networks. In particular we identify redundant connections and illustrate how ill-conditioning may otherwise arise, which motivates the use of regularization.

Having acknowledged the need for regularization makes way for the highly effective second-order methods for training. In this contribution we particulary focus on the damped Gauss-Newton method and illustrate how this method by far outperforms gradient descent on a time series prediction problem, namely the Santa Fe laser data. The focus in this contribution is on time series prediction, but the results generalize to other applications as well.

\section{ARCHITECTURE}

The general architecture of the networks considered here are fully connected feedback networks with one hidden layer of nonlinear units and a single linear 
output unit. The output $y(t)$ of the network is linear in order to allow for arbitrary dynamical range, and is given by

$$
y(t)=\sum_{i=1}^{N_{h}} w_{o i} s_{i}(t)+w_{o b}
$$

where $N_{h}$ is the number of hidden units, $w_{o i}$ is the weight to the output unit from hidden unit $j$ and $w_{o b}$ is a bias weight. The output $s_{i}(t)$ from hidden unit $i$ at time $t$ is computed as

$$
s_{i}(t)=f\left(\sum_{j=1}^{N_{h}} w_{i j} s_{j}(t-1)+w_{i o} y(t-1)+\sum_{k=1}^{N_{I}} w_{i k} x_{k}(t)+w_{i b}\right)
$$

where $w_{i j}$ is the weight to hidden unit $i$ from hidden unit $j, w_{i o}$ is the weight to hidden unit $i$ from the output unit and $w_{i b}$ is the bias weight for hidden unit $i . x_{k}(t)$ is the $k$ 'th element in the external input vector $\mathbf{x}(t)$ at time $t$ and $N_{I}$ is the total number of external inputs. $f(\cdot)$ is the nonlinear activation function, in this work we use $f(x)=\tanh (x)$.

Note that the update of the recurrent network presented above is layered, as the outputs $s_{i}(t)$ from the hidden units are computed immediately before the computation of the output unit output. This is opposed to the update presented in e.g. [10] where all the units are updated simultaneously. In [6] it was shown that when using fully recurrent networks for forecasting, layered update is preferable since synchronous update of the units effectively results in a two-step ahead predictor. Note also that the linear output unit does not have feedback of its own previous value. This is in order to avoid stability problems that are otherwise likely to occur.

\section{Training}

In this work we focus on time series prediction in which case the input vector contains delayed elements of the time series, $\mathbf{x}(t)=\left[x(t), \ldots, x\left(t-N_{I}+1\right)\right]$, and the network output is a prediction of the next value in the series, $\widehat{x}(t+1)=$ $y(t)$. Training the network means adjusting the weights so as to minimize a cost function. Most applications are based on the sum of squared errors,

$$
E(\mathbf{w})=\frac{1}{2} \sum_{t=1}^{T}[e(t)]^{2}, e(t)=x(t+1)-y(t)
$$

where $T$ denotes the number of training examples and $\mathbf{w}$ is the concatenated set of parameters. The adjustment of the parameters is done off line by an iterative sheme, $\mathbf{w}_{k+1}=\mathbf{w}_{k}-\eta \Delta \mathbf{w}_{k}$, where $\Delta \mathbf{w}_{k}$ indicates the direction of change and $\eta$ is the (adaptive) size of the step. When training recurrent neural networks the most commonly used scheme is gradient descent, where the direction $\Delta \mathbf{w}_{k}$ is equal to the gradient $\mathbf{g}, g_{i}=\partial E\left(\mathbf{w}_{k}\right) / \partial w_{i}$. Unfortunately this method suffers from extremely slow convergence, and the quality of resulting solutions is often not satisfactory. 
Experiments have shown that much more efficient training can be obtained by using second-order methods [6]. Here we focus on the damped GaussNewton method [3], in which the search direction $\Delta \mathbf{w}_{k}$ is determined by

$$
\Delta \mathbf{w}_{k}=\mathbf{H}^{-1} \mathbf{g}
$$

where $\mathbf{H}$ is the positive semidefinite approximation to the Hessian,

$$
H_{i j}=\sum_{t=1}^{T} \frac{\partial y(t)}{\partial w_{i}} \frac{\partial y(t)}{\partial w_{j}} \approx \frac{\partial^{2} E\left(\mathbf{w}_{k}\right)}{\partial w_{i} \partial w_{j}}=\sum_{t=1}^{T}\left[\frac{\partial y(t)}{\partial w_{i}} \frac{\partial y(t)}{\partial w_{j}}-e(t) \frac{\partial^{2} y(t)}{\partial w_{i} \partial w_{j}}\right]
$$

In each iteration $k$ the step size $\eta$ is determined by line search which makes the method globally convergent [3]; here we recommend a simple approach where $\eta$ is halved until a decrease in the cost is obtained [3]. The iterations are continued until convergence, determined by a sufficiently small length of the gradient, $\|\mathbf{g}\|_{2}<\varepsilon$. The Gauss-Newton method involves finding the solution to a linear system of equations $\mathbf{H} \Delta \mathbf{w}_{k}=\mathbf{g}$ in each iteration, but the increased computational burden is justified by a dramatic increase in convergence and thus reduction of overall training time, even for large networks as we shall see. However, the success of the damped Gauss-Newton method relies heavily on the conditioning of the training problem, as is the case for gradient descent.

\section{ILL-CONDITIONING}

When training using either gradient descent or the Gauss-Newton method, a measure of great importance for the convergence is the condition number of the Hessian $\mathbf{H}$. For a symmetric positive definite matrix $\mathbf{H}$, the condition number is defined as $\kappa(\mathbf{H})=\lambda_{\max } / \lambda_{\min }$, the ratio between the largest and smallest eigenvalue of $\mathbf{H}$. If the condition number is large, the Hessian becomes ill-conditioned. The convergence rate will suffer and the solution to the linear system of equations (4) in the Gauss-Newton method becomes unreliable. As a rule of thumb the solution may not be trustworthy if $\kappa(\mathbf{H})>\epsilon^{-1 / 2}$, where $\epsilon$ denotes the machine precision [3]. For the IEEE 64-bit floating point representation this is equivalent to $\kappa(\mathbf{H})>6.7 \cdot 10^{7}$. This may seem as a large number, but this order of magnitude is not uncommon in the framework of either feedforward networks [7] or recurrent networks as we shall see.

In [2] it was shown that an eigenvalue of the order of the number of input variables could be avoided if the mean was subtracted from each of the input variables $x_{k}(t)$ and if a symmetric activation function is used. However, these simple countermeasures are not adequate for avoiding ill-conditioning in recurrent networks, as the analysis in the following will show.

The Hessian (5) can also be written as

$$
\mathbf{H}=\mathbf{J}^{\mathrm{T}} \mathbf{J}, J_{t i}=\frac{\partial y(t)}{\partial w_{i}}
$$

where $\mathbf{J}$ is the Jacobian matrix, whose columns are the partial derivatives of the network output at each timestep in the training series. If $\mathbf{J}$ is rankdeficient some of the columns are linearly dependent, which is indicated by 
singular values with the value zero in an SVD analysis. This again leads to a singular Hessian and thus an infinite condition number. In practice it is rare to find columns in $\mathbf{J}$ that are exactly dependent and thus singular values that are exactly zero [7]. However, it is often the case that columns are nearly linearly dependent, which leads to very small singular values of $\mathbf{J}$ and thus large condition numbers for the Hessian $\mathbf{H}$. In the following sections we describe situations leading to ill-conditioning of $\mathbf{J}$ for recurrent networks, arising from both exact and approximate linearly dependencies between columns in $\mathbf{J}$.

\section{Exact dependency}

For the type of recurrent networks defined by (1) and (2) there is built-in rank deficiency in the Jacobian since it is easy to show that some of the columns in $\mathbf{J}$ will always be linear combinations of each other. This is illustrated by an example for a small network, but the result apply for networks with an arbitrary number of hidden units. The network considered here involves only one external input and one hidden unit, and the output is thus defined as

$$
\begin{aligned}
y(t) & =w_{o 1} s_{1}(t)+w_{o b} \\
s_{1}(t) & =f\left(w_{11} s_{1}(t-1)+w_{1 o} y(t-1)+w_{1 x} x(t)+w_{1 b}\right) \\
& =f\left(\left(w_{11}+w_{1 o} w_{o 1}\right) s_{1}(t-1)+w_{1 x} x(t)+\left(w_{1 b}+w_{1 o} w_{o b}\right)\right)
\end{aligned}
$$

where (9) is obtained by insertion of (7) in (8). We see that the network output will remain unchanged as long as the total weighting $k_{1}$ of $s_{1}(t-1)$, $k_{1}=w_{11}+w_{1 o} w_{o 1}$, and the total bias $k_{2}$ on the hidden unit, $k_{2}=w_{1 b}+$ $w_{1 o} w_{o b}$, remains constant. $w_{o 1}$ and $w_{o b}$ can not be changed without directly affecting the network output (7) and are therefore kept fixed which we denote by $*$. However, changes in $w_{11}, w_{10}$ and $w_{16}$ that satisfies both expressions

$$
\begin{gathered}
w_{11}+w_{o 1}^{*} \cdot w_{1 o}+0=k_{1} \\
0+w_{o b}^{*} \cdot w_{1 o}+w_{1 b}=k_{2}
\end{gathered}
$$

will leave the network output unchanged. The expressions (10) form hyperplanes in parameter space spanned by $w_{11}, w_{10}$ and $w_{1 b}$ and their line of intersection is computed as $\left(w_{11}, w_{1 o}, w_{1 b}\right)=\left(k_{1}, 0, k_{2}\right)+t\left(-w_{o 1}^{*}, 1,-w_{o b}^{*}\right)$, parametrized by $t$. The line defines a direction in parameter space in which the network output is constant. The constant network output means that derivatives are zero in this direction. Thus, columns in the Jacobian corresponding to $\left(w_{11}, w_{1 o}, w_{1 b}\right)$ are linearly dependent.

When investigating Jacobians for the dependency problem outlined above it is however uncommon to encounter singular values exactly equal to zero; but according to the derivations this clearly ought to be the case. The reason for this is the initialization of previous state values when starting up the network. If the recurrent network starts iteration at time $t=1$ it is common practice [10] to set the previous states of the hidden units as well as their derivatives to zero, $s_{i}(0)=0, \partial s_{i}(0) / \partial \mathbf{w}=0$. This startup procedure clearly marks an initial discontinuity in the recursive equations (7) and (8) 
governing the feedback network. Thus initially the partial derivatives wrt. the involved weights in the Jacobian will generally not be linearly dependent. But after a few iterations indicating a transient, the dependency arises with increasing accuracy. The linear dependency is eliminated if we omit the feedback weights $w_{i o}$ from the output to the hidden units $i$, as the degeneracy can then no longer occur. This elimination has no influence on the modeling capabilities of the network since the remaining weights can be adjusted so that the network output remains unaffected.

\section{Approximate dependency}

Even though removal of the feedback weights $w_{i o}$ leading from the linear output to the hidden units removes the problem of almost exact rank-deficiency in the Jacobian for recurrent networks it does not eliminate ill-conditioning as experiments show. In [7] the problem of ill-conditioning was analyzed for feedforward networks by careful examination of the components entering the partial derivatives $\partial y(t) / \partial w_{i}$ of the network output and it was found that ill-conditioning in the Jacobian can arise from at least these three reasons (assuming that the external inputs are not proportional):

1. The output from a hidden unit is saturated and constant $(\equiv \pm 1)$.

2. The outputs from two hidden units are approximately proportional.

3. The derivatives of two hidden unit outputs wrt. their activations are approximately proportional.

Theoretical and empirical examinations of the components entering the partial derivatives for recurrent networks reveal that ill-conditioning may arise here from the same reasons; such analysis is however not included here.

Situation 2 where the outputs of two hidden units are proportional and thus highly correlated often occurs in practice; e.g., in [9] high correlation between hidden unit outputs was found and studied for feedforward networks.

The effects of situation 2 are similar to the effects of exact dependencies described above, as we can determine directions in parameter space in which the cost function is approximately constant. For recurrent networks this situation is much more severe than for feedforward networks since the degeneracy will not only affect weights leading to the output, but also many weights connecting the hidden units as the experiments will show.

The scenarios listed above lead to nearly linearly dependencies between the columns of $\mathbf{J}$ and thus to small eigenvalues in $\mathbf{H}$. However, the condition number of a matrix is determined by the ratio between the largest and smallest eigenvalues, thus problems do not only arise from small singular values but also from large values. As mentioned, the situations described above will lead to directions in parameter space where the cost is approximately constant, thus when training using the Gauss-Newton method the search direction will be dominated by these directions leading to an unrestrained growth in the magnitude of the affected weights. This again leads to a significant growth in 
the magnitude of several of the columns in the Jacobian since many derivatives are dominated by terms of the form [10]

$$
\frac{\partial s_{k}(t)}{\partial w_{p q}} \propto \sum_{j=1}^{N_{h}} w_{k j} \frac{\partial s_{j}(t-1)}{\partial w_{p q}}
$$

which becomes large if the weights $w_{k j}$ become large. The large elements in the Jacobian lead to an overall upward scale of the elements in $\mathbf{J}^{\mathrm{T}} \mathbf{J}$ and thus to an upward shift of the eigenvalues.

\section{REGULARIZATION}

A traditional method for handling ill-conditioning is by regularizing the cost function $[3,4]$. A simple yet highly effective regularization can be obtained by augmenting the cost function by a simple quadratic weight decay [4],

$$
C(\mathbf{w})=E(\mathbf{w})+\frac{\alpha}{2} \mathbf{w}^{\mathrm{T}} \mathbf{w}
$$

Simple weight decay is often primarily considered as a means for avoiding overfitting as it puts constraints on the parameters and thus reduces the degrees of freedom. Weight decay should however also be considered from its regularizing effects. The immediate effect is that $\alpha$ gets added to the diagonal of the Hessian which puts a lower bound on the smallest eigenvalues, since it is easy to show that $\lambda(\mathbf{H}+\alpha \mathbf{1})=\lambda(\mathbf{H})+\alpha$. Another effect is the limit imposed on the growth of the weights which prevents near singular directions in parameter space from dominating the search directions obtained by the Gauss-Newton method, thus greatly improving the efficiency of the optimization. The constraints put on the weights by the regularization has a smoothing effect on the cost function which was clearly illustrated in [6]. Here it was also demonstrated that the significance of the second order term ignored in (5) diminishes when using simple weight decay as regularization.

\section{EXPERIMENTS}

In the first experiment we illustrate how ill-conditioning results from some of the situations described herein and how regularization improves training. For this experiment we used a simple recurrent network to predict the laser data from the Santa Fe time series prediction competition [8]. The data were scaled so that the first 1000 points used for training had zero mean and unit variance and the following 100 values were used as a test set. The network used had one external input and three hidden units; there were no feedback from the linear output unit to the hidden units as found appropriate above. Training was performed initially using five iterations of gradient descent followed by the damped Gauss-Newton method. In the left panel of Figure 1 is shown the evolution of the mean squared errors normalized by the variance of the sets (NMSE, [8]) when training without regularization. It seems that training is converging to a solution, but this is not the case as the evolution of the weights 

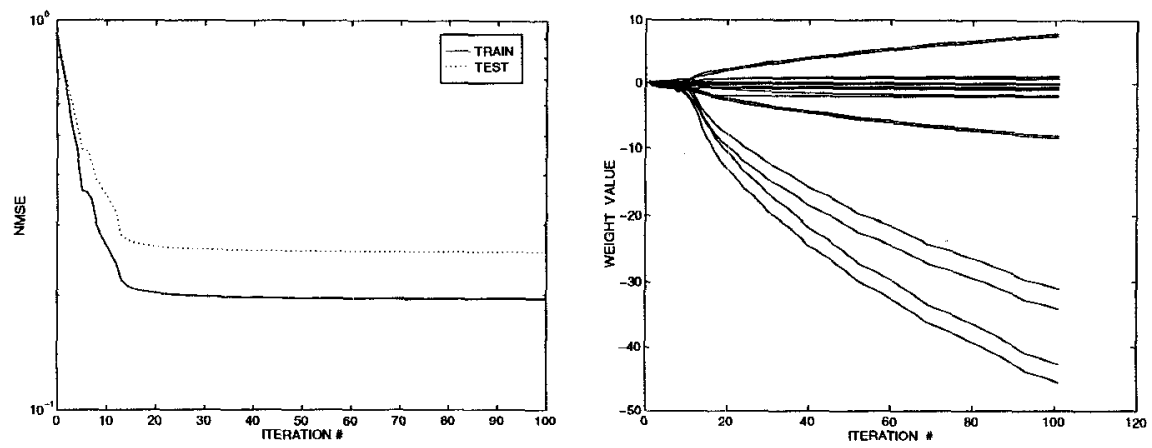

Figure 1: Training without regularization. Left panel: Evolution of training and test errors. Right panel: Evolution of the weight values.

in the right panel of Figure 1 shows. What happens is that the outputs of two hidden units become almost proportional; this is revealed by the cosine to the angle $\theta$ between vectors containing their outputs on the training set which at iteration 100 is $\cos \theta=0.9998$. This corresponds to situation 2 listed above. The weights that grow in magnitude are the pairs of weights leading from these two units to every unit in the network including the output. Note that the error and thus the network output is unaffected since the effects of the changes in the growing weights cancel out due to the dependency between the hidden units.

The condition number during training is shown in the left panel of Figure 2 and is seen to grow enormously. The rapid increase occurs shortly after the initiation of the second-order method which quickly 'discovers' the dependency between the hidden unit outputs. The near singular Hessian $\mathbf{H}$ leads to very large weight changes in some directions when solving (4). The large steps are however handled by the line search which returns very small step sizes, indicated by the smooth increase in the weight magnitudes. In the right panel of Figure 2 is shown the eigenvalues of the Hessian after iterations 7, 20 and 100. At each of the iterations it is seen that the condition number results from both very small as well as very large eigenvalues and we note that as
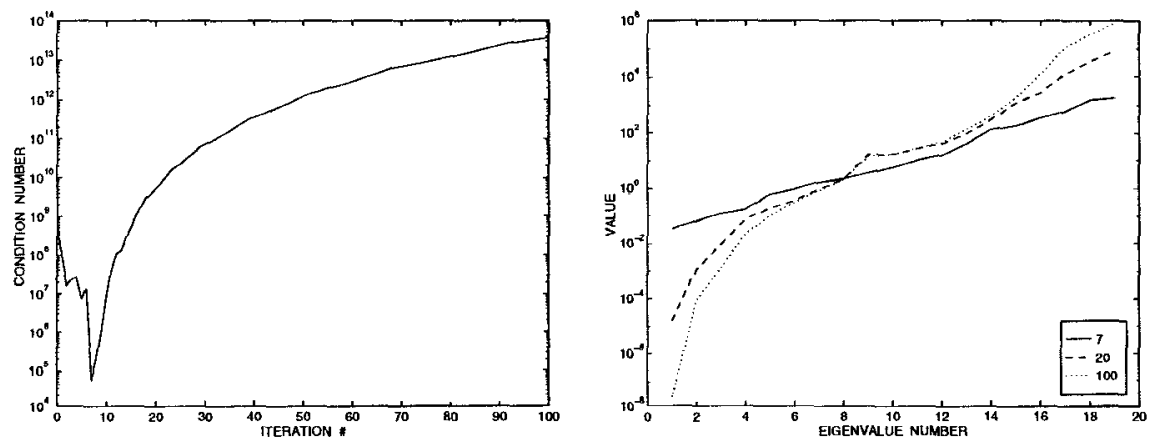

Figure 2: Training without regularization. Left panel: Evolution of the condition number for $\mathbf{H}$. Right panel: Eigenvalues after iterations 7, 20 and 100 . 

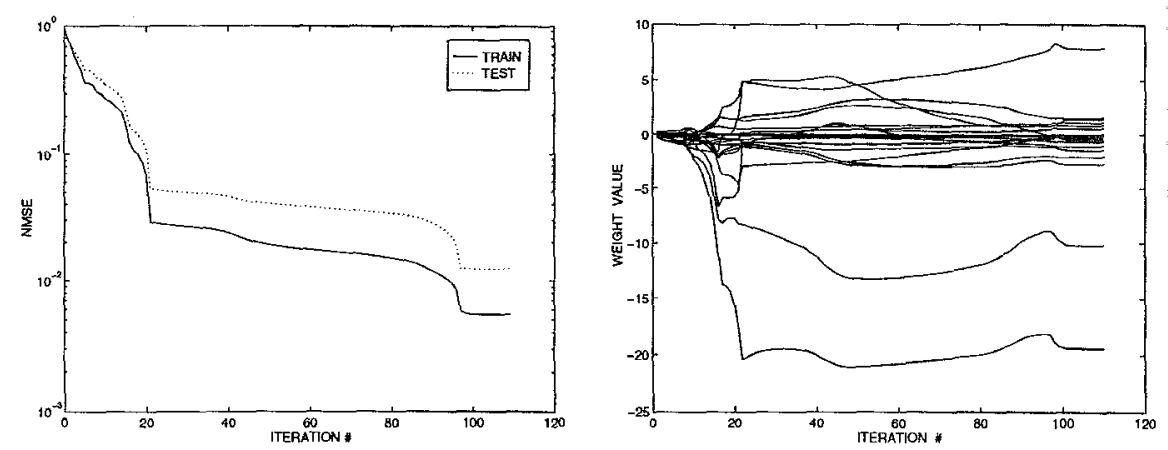

Figure 3: Training with regularization, $\alpha=10^{-3}$. Left panel: Evolution of training and test errors. Right panel: Evolution of the weight values.

training progresses the eigenvalues extend both upward and downward.

The training was then repeated using the exact same initial weights and the same training approach, but now with a regularization term added to the cost function, using $\alpha=10^{-3}$. In the left panel of Figure 3 is shown the resulting evolution of the errors. The positive effect of the regularization is evident, as the final errors are several orders of magnitude below the levels shown in Figure 1 obtained without regularization. Furthermore the stopping criterion $\|\mathbf{g}\|_{2}<10^{-4}$ was satisfied; in the previous experiment using no regularization the gradient norm grew proportional to the condition number.

In the right panel of Figure 3 we see that the regularization term limits the growth of the weights compared to Figure 1. Some however still grow large as does the condition number shown in the left panel of Figure 4. Even though the condition number grows to $10^{8}$ the damped Gauss-Newton method still manages to find a minimum. Experience shows that for this method successful training to a (local) minimum can be obtained for condition numbers up to about $10^{8}$ in magnitude. This may depend on the decomposition algorithm used when solving (4), here we use the fast and stable Cholesky factorization [3]. From the right panel of Figure 4 we learn that the reduction in condition number is obtained only from an increase in the smallest eigenvalues resulting
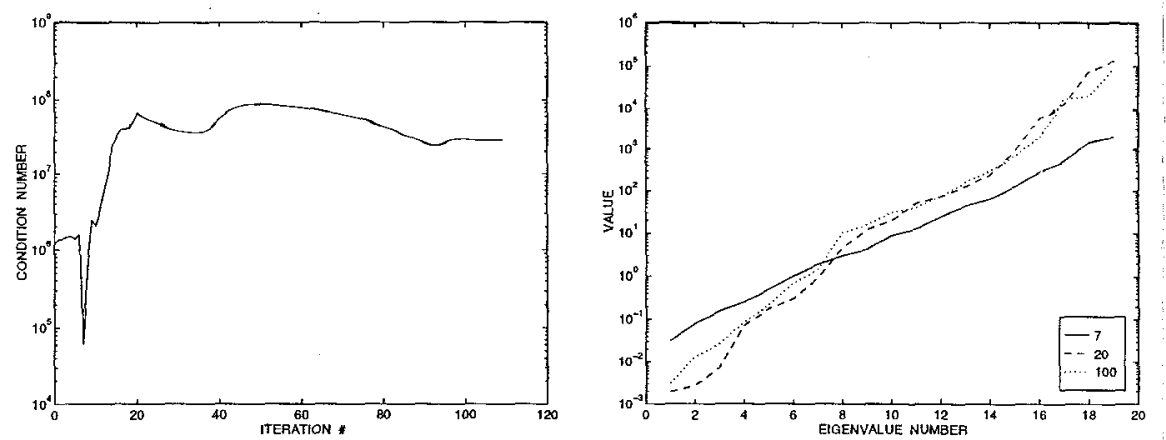

Figure 4: Training with regularization, $\alpha=10^{-3}$. Left panel: Evolution of the condition number for $\mathbf{H}$. Right panel: Eigenvalues after iterations 7, 20 and 100 . 
from the regularization. The largest eigenvalues are of the same order of magnitude as when training without weight decay, see Figure 2. This is due to the still fairly large weight magnitudes. If the regularization term $\alpha$ is further increased the larger eigenvalues will also be affected; but so will the modeling capabilities of the network, leading to increased errors.

In the final experiment we compare the performance of damped GaussNewton with a gradient descent algorithm also using the step-size halving line search. The problem is still prediction of the laser series but using larger networks with a single input and nine hidden units, 109 weights in total (no feedback from the output to the hidden units). Thus, each iteration using damped Gauss-Newton involved solution of a 109 by 109 linear system of equations. Six initial networks were generated by initializing their weights with values drawn from a uniform distribution over the interval $[-0.3 ; 0.3]$. The training algorithms were then compared when starting from the same six initial networks, both using regularization $\alpha=0.02$. The resulting evolution of errors is shown in Figure 5; in the left panel we see the resulting errors using the damped Gauss-Newton method, in the right panel using gradient descent. Using both methods the stopping criteria was set to $\|\mathbf{g}\|_{2}<10^{-4}$ or maximum 10000 iterations.
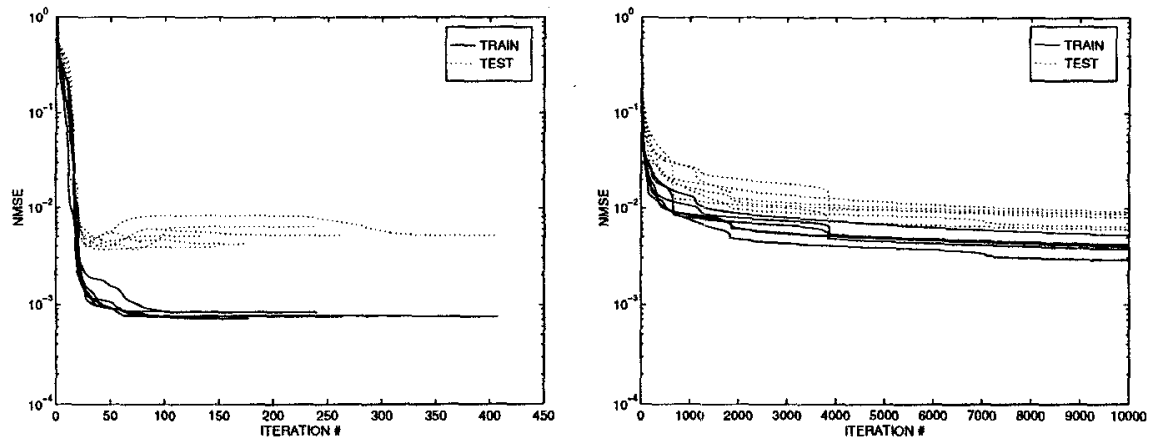

Figure 5: Evolution of errors using different optimization methods. Left panel: Damped Gauss-Newton method. Right panel: Gradient descent with line search.

For the damped Gauss-Newton method the stopping criterion was met in all six runs. The average training error (Normalized Mean Squared Error) was $7.7 \cdot 10^{-4}$, the average test error was $4.9 \cdot 10^{-3}$. The average time for a complete training run was 200 seconds. For gradient descent the stopping criterion was never met, the termination of the algorithm in each run was due to maximum number of iterations reached. The average training error obtained after the maximum allowed 10000 iterations was $4.0 \cdot 10^{-3}$, the average test error was $7.8 \cdot 10^{-3}$. The average time used for obtaining these error levels was 8140 seconds. Note that the levels of both training and test errors obtained using gradient descent are much higher than the levels obtained using the damped Gauss-Newton method even though gradient descent used a factor of 50 times more iterations and a factor of $\mathbf{4 0}$ times more computer time. Thus, even though an iteration of the damped Gauss-Newton method is computationally 
more costly than an iteration of gradient descent, the additional cost is highly justified by the vastly increased convergence rate. Similar justification has been observed for networks with up to 300 parameters.

\section{CONCLUSION}

In this paper we have focused on sources of ill-conditioning and thus the need for regularization when training recurrent networks especially using secondorder methods. Once this need is recognized dramatic improvement in convergence rate and quality of solution is obtained, even for large size problems.

\section{ACKNOWLEDGMENTS}

The author would like to thank Lars Kai Hansen and Jan Larsen for support. This research is supported by the Danish Natural and Technical Research Coucils through the Computational Neural Network Center (CONNECT).

\section{REFERENCES}

[1] Y. Bengio, P. Simard and P. Frasconi, "Learning long-term dependencies with gradient descent is difficult," IEEE Transactions on Neural Networks, vol. 5, no. 2, pp. 157-166, 1994.

[2] Y. L. Cun, I. Kanter and S. A. Solla, "Eigenvalues of covariance matrices: Application to neural-network learning," Physical Review Letters, vol. 66, no. 18, pp. 2396-2399, 1991.

[3] J. E. Dennis and R. B. Schnabel, Numerical Methods for Unconstrained Optimization and Nonlinear Equations, Englewood Cliffs, NJ: PrenticeHall, 1983.

[4] S. Haykin, Neural Networks, A Comprehensive Foundation, New York, NY: Macmillan, 1994.

[5] S. Hochreiter and J. Schmidhuber, "Long short term memory," Tech. Rep. FKI207-95, Fakultat fur Informatik, Technische Universitat Munchen, Munchen, 1995.

[6] M. W. Pedersen and L. K. Hansen, "Recurrent networks: Second order properties and pruning," in G. Tesauro, D. Touretzky and T. Leen, eds., Advances in Neural Information Processing Systems, The MIT Press, 1995, vol. 7, pp. 673-680.

[7] S. Saarinen, R. Bramley and G. Cybenko, "Ill-conditioning in neural network training problems," SIAM Journal on Scientific Computing, vol. 14, pp. $693-714,1993$.

[8] A. S. Weigend and N. A. Gershenfeld, eds., Time Series Prediction: Forecasting the Future and Understanding the Past, Reading, MA: AddisonWesley, 1993.

[9] A. S. Weigend and D. E. Rumelhart, "The effective dimension of the space of hidden units," in E. Keramides, ed., Proceedings of INTERFACE'91: Computing Science and Statistics, Springer Verlag, 1992.

[10] R. J. Williams and D. Zipser, "A learning algorithm for continually running fully recurrent neural networks," Neural Computation, vol. 1, pp. 270-280, 1989. 